\title{
Los suplementos de calcio ¿aumentan el riesgo de eventos cardiovasculares?
}

Do the calcium supplements increase the risk of cardiovascular events?

Bolland MJ,y col. Bmj. 2010;341:c3691.

\section{Objetivo}

Determinar si el uso de suplementos de calcio aumentan el riesgo de eventos cardiovasculares.

\section{Diseño}

Revisión sistemática y meta-análisis a nivel de ensayo y de pacientes.

\section{Fuentes de datos y selección de los estudios}

Medline, Embase, Registro Cochrane de Ensayos Controlados, listas de referencias y registros de ensayos clínicos (1966 a 2010). Se incluyeron ensayos clínicos aleatorizados (ECA) controlados con placebo que evaluaran el uso de suplementos de calcio ( $\geq 500 \mathrm{mg} / \mathrm{día}$ ), de duración superior a un año, con 100 o más participantes mayores de 40 años.

\section{Extracción de datos}

La selección, evaluación crítica y extracción de los datos fue realizada de manera independiente por dos revisores. Los autores principales de los ensayos suministraron los datos necesarios. Los eventos cardiovasculares se obtuvieron mediante auto-reporte, ingresos hospitalarios o certificados de defunción.

\section{Resultados}

De 15 ensayos elegibles, 11 reportaron resultados (11.921 participantes, seguimiento medio de cuatro años) y cinco de estos últimos aportaron datos a nivel paciente (8.151 participantes, seguimiento mediano 3,6 años). Los resultados se presentan en la tabla 1.

Tabla 1. Número de eventos por rama y riesgo relativo a nivel paciente y ensayo

\begin{tabular}{|c|c|c|c|c|c|c|}
\hline \multirow{2}{*}{$\begin{array}{c}\text { Resultado } \\
\text { Infarto de miocardio }\end{array}$} & \multirow{2}{*}{$\begin{array}{c}\text { Nivel } \\
\text { (Estudios; N calcio / N Placebo) } \\
\text { Paciente }(5 ; 4.097 / 4.054) \\
\text { Ensayo }(7 ; 5.205 / 5.005) \\
\end{array}$} & \multicolumn{2}{|c|}{ Calcio (\%) } & \multicolumn{2}{|c|}{ Placebo (\%) } & RR (IC95\%) \\
\hline & & $\begin{array}{l}143 \\
166\end{array}$ & $\begin{array}{l}(3.5 \%) \\
(3.2 \%)\end{array}$ & \multicolumn{2}{|c|}{\begin{tabular}{l|l}
1111 & $((2.7 \%)$ \\
130 & $(2.6 \%)$ \\
\end{tabular}} & $\begin{array}{l}1,31(1,02 \text { a } 1,67) \\
1,27(1,01 \text { a } 1,59)\end{array}$ \\
\hline ACV & $\begin{array}{c}\text { Paciente }(5 ; 4.097 / 4.054) \\
\text { Ensayo }(8 ; 5.339 / 5.246)\end{array}$ & $\begin{array}{l}190 \\
212\end{array}$ & $\begin{array}{l}(4.6 \%) \\
(4.0 \%)\end{array}$ & $\begin{array}{l}156 \\
190\end{array}$ & $\begin{array}{l}(3.8 \%) \\
(3.6 \%)\end{array}$ & $\begin{array}{l}1,24(0,99 \text { a } 1,56) \\
1,12(0,92 \text { a } 1,36)\end{array}$ \\
\hline Infarto, ACV o muerte súbita & $\begin{array}{c}\text { Paciente }(5 ; 4.097 / 4.054) \\
\text { Ensayo }(8 ; 5.273 / 5.072)\end{array}$ & $\begin{array}{l}361 \\
358\end{array}$ & $\begin{array}{l}(8.8 \%) \\
(6.8 \%)\end{array}$ & $\begin{array}{l}287 \\
319\end{array}$ & $\begin{array}{l}(7.1 \%) \\
(6.3 \%)\end{array}$ & $\begin{array}{l}1,27(1,07 \text { a } 1,51) \\
1,12(0,97 \text { a } 1,30)\end{array}$ \\
\hline Muerte & $\begin{array}{l}\text { Paciente }(5 ; 4.097 / 4.054) \\
\text { Ensayo }(7 ; 5.041 / 4.998)\end{array}$ & $\begin{array}{l}519 \\
559\end{array}$ & $\begin{array}{l}(12.7 \%) \\
(11.1 \%)\end{array}$ & $\begin{array}{l}487 \\
535\end{array}$ & $\begin{array}{l}(12.0 \%) \\
(10.7 \%)\end{array}$ & $\begin{array}{l}1,09(0,96 \text { a } 1,23) \\
1,07(0,95 \text { a } 1,19)\end{array}$ \\
\hline
\end{tabular}

ACV: Accidente cerebrovascular. IC95\%: intervalo de confianza del 95\%.

\section{Conclusiones}

El uso de suplementos de calcio (sin vitamina D) se asocia con un mayor riesgo de infarto de miocardio (IM).

\section{Objetivo}

Determinar si el uso de suplementos de calcio aumenta la mortalidad por cualquier causa o la enfermedad coronaria.

\section{Diseño}

Revisión sistemática y meta-análisis con datos de pacientes individuales.

\section{Fuentes de datos y selección de estudios}

Medline (1966 a 2013), Embase (1974 a 2013), Registro Cochrane de Ensayos Controlados (1970 a 2013), listas de re-ferencias y referencias de ensayos clínicos (1966 a 2013). Se incluyeron ECA controlados con placebo que evaluaran el uso de suplementos de calcio ( $\geq 500 \mathrm{mg} /$ día), de duración superior a un año, con mujeres de 50 años o más, sin límites de tamaño muestral.

Tabla 2. Número de eventos por rama y riesgo relativo

\begin{tabular}{c|l|c|c|c}
\multirow{2}{*}{\begin{tabular}{c} 
Resultado \\
\multirow{2}{*}{ Resultados primarios }
\end{tabular}} & Calcio (\%) & Placebo (\%) & RR (IC95\%) \\
\cline { 2 - 5 } & Enfermedad cardiovascular (5; 24.284/24.176) & $1.720(7.1 \%)$ & $1.670(6.9 \%)$ & $1,02(0,96 \mathrm{a} 1,09)$ \\
\hline \multirow{2}{*}{ Resultados Secundarios } & Muerte $(17 ; 31.108 / 31.275)$ & $2.053(6.6 \%)$ & $2.104(6.7 \%)$ & $0,96(0,91 \mathrm{a} 1,02)$ \\
\cline { 2 - 5 } & Infarto de miocardio $(7 ; 25.908 / 25.203)$ & $584(2.2 \%)$ & $539(2.1 \%)$ & $1,08(0,93 \mathrm{a} 1,25)$ \\
\cline { 2 - 5 } & Angor/Síndrome Coronario agudo $(4 ; 24.145 / 23.888)$ & $458(1.9 \%)$ & $418(1.7 \%)$ & $1,09(0,95 \mathrm{a} 1,24)$ \\
\cline { 2 - 5 } & Enfermedad coronaria crónica $(4 ; 24.145 / 23.888)$ & $774(3.2 \%)$ & $732(3.2 \%)$ & $0,92(0,73 \mathrm{a} 1,15)$ \\
\hline
\end{tabular}

IC95\%: intervalo de confianza.

\section{Conclusiones}

Los suplementos de calcio (con o sin vitamina D) no se asocian
Fuente de financiamiento: Health Research Council of New Zealand and the University of Auckland School of Medicine Foundation.

Lewis JR, y col. Journal of Bone and Mineral Research. 2015;30(1):165-175

\section{Extracción de datos}

La selección, evaluación crítica y extracción de los datos fue realizada de manera independiente por dos revisores. Estos contactaron a los autores principales de los ensayos para verificar su elegibilidad. Los disensos fueron resueltos mediante discusión hasta alcanzar un acuerdo. La evaluación de calidad fue realizada usando la herramienta de la colaboración Cochrane para evaluar riesgo de sesgos. Los eventos cardiovasculares fueron clasificados mediante las guías de reporte de la Asociación Americana del Corazón usando los códigos CIE 10. Solo se incluyeron estudios que reportaban resultados ve-rificados por revisión clínica, ingresos hospitalarios o certificados de defunción.

\section{Resultados}

De 17 ensayos elegibles, cinco reportaron resultados de enfermedad cardiovascular y 17 reportaron resultados de mortalidad. Los resultados presentaron baja heterogeneidad (ver tabla 2). 


\section{Comentario}

La revisión sistemática de Bolland y col. fue motivada por la observación de un posible incremento de eventos cardiovasculares en un ensayo clínico que evaluó el uso de suplementos cálcicos en mujeres mayores saludables ${ }^{1,2}$. Su meta-análisis encontró que sólo uno (IM) de los cuatro resultados evaluados resultó estadísticamente significativo, aunque con un incremento de solo un $1 \%$ en el extremo inferior del IC 95\%, lo cual cuestiona su relevancia clínica. Sin embargo los autores dedujeron que esto podría implicar una gran carga de enfermedad en la población por lo cual sugirieron reevaluar el rol de los suplementos cálcicos en el manejo de la osteoporosis. Esta conclusión es cuanto menos temeraria por razones de validez interna y externa que se detallan a continuación: 1) El cuerpo de la evidencia resulta parcial, debido a la exclusión de estudios pequeños y la existencia de datos incompletos (sobre los 15 ensayos, datos sobre resultados cardiovasculares disponibles para el $63 \%$ de los participantes a nivel paciente y para el $85 \%$ a nivel ensayo). 2) Problemas metodológicos de los ECA incluidos: la asignación enmascarada sólo fue descripta en cuatro estudios y la aleatorización en ocho. Esto podría haber redundado en asimetrías en la distribución de factores de riesgo (por ejemplo, mayor nivel de LDLc en el grupo expuesto a suplementos de calcio de un ECA incluido'), y aún más influyente resultaría una asimetría en la historia de cardiopatía isquémica, cuyos datos sólo se obtuvieron en cuatro de los $11 \mathrm{ECA}^{3}$. Excepcionalmente existió cegamiento de los evaluadores (importante para IM y accidente cerebrovascular), la recolección de datos no siguió un protocolo pues los estudios perseguían resultados no cardiovasculares (ej. Osteoporosis) y en por lo menos siete de los ECA la adjudicación retrospectiva de resultados se basó en auto-reportes.

El estudio de Hsia el al. ${ }^{3}$, ECA que evaluó el efecto de la suplementación con calcio/vitamina $D$ en la ocurrencia de eventos cardiovasculares (definición pre-especificada de resultado, con asignación centralizada prospectiva y 254.000 pacientes-año de seguimiento), no halló aumento del riesgo de IM, incluso en el subgrupo con similares características a los del meta-análisis de Bolland (RR de IM 0,9; IC95\% 0,55 a 1,48). La evaluación de vitamina $\mathrm{D}$ excluyó a este estudio del meta-análisis, pero difícilmente explique la totalidad de las diferencias.

Aplicando el abordaje GRADE ${ }^{4}$, el meta-análisis de Bolland y col. presenta baja calidad de evidencia, por los problemas metodológicos y la imprecisión de sus resultados dada por la amplitud de sus IC95\%. La baja calidad de evidencia implica una alta probabilidad que nueva evidencia modifique la estimación del efecto. Incluso podría ser de muy baja evidencia si jerarquizamos lo incompleto del cuerpo de la evidencia o se calificara como muy serio el riesgo de sesgo.

El recientemente publicado estudio de Lewis y col., que aquí también resumimos, incluyó estudios aleatorizados que reportaran eventos cardiovasculares clínicamente verificados por registros de hospitales o certificados de defunción (a diferencia de la revisión de Bolland y col., que en muchos casos se basó en auto-reportes). Esta nueva revisión, constituye el mayor grado de evidencia posible en favor de la seguridad de los suplementos de calcio, por su alta calidad (sistema GRADE) y por haber realizado un metanálisis de datos de pacientes individuales sin encontrar evidencia de que estos incrementaran el riesgo cardiovascular o la mortalidad. Por otro lado, en ninguna de las revisiones se mencionan estudios observacionales de efectos negativos del enriquecimiento alimentario del calcio. Bolland y col. argumentan que el hiperparatiroidismo primario se asocia con eventos cardiovasculares pero en realidad la suplementación con calcio en todo caso lo previene. Por el contario, nosotros demostramos fuerte evidencia de que el calcio reduce ligeramente la presión arterial, lo que contrastaría con el presunto incremento del riesgo cardiovascular. ${ }^{5}$

Bolland y col. sugieren también, en base a sus hallazgos, que debería revisarse el rol de los suplementos de calcio en la prevención de la osteoporosis sin discriminar edad. Sin embargo ambas revisiones estudiaron mujeres de edad avanzada con alta ingesta basal de calcio (media de $860 \mathrm{mg}$ de calcio por día) que sumado a la suplementación de calcio recibida (entre 500 y $2.000 \mathrm{mg}$ por día, media de $1.200 \mathrm{mg} /$ día) superaban holgadamente las recomendaciones de diferentes guías (de 1.000 a $1.300 \mathrm{mg} /$ día), acercándose al límite superior de lo recomendado (2.000 mg/día). A pesar de que la dosis total recibida es mucho mayor que la utilizada por la población, Lewis y col. no encontraron un efecto deletéreo de los suplementos de calcio.

Conclusiones de los comentadores

El meta-análisis de Bolland y col. plantea una hipótesis inquietante. La baja calidad de la evidencia que provee, de ninguna manera justifica replantear las recomendaciones preventivas de aportes de calcio, sin embargo la difusión de estudios realizados en Nueva Zelanda, donde lo advertían, produjo una caída del $66 \%$ de las prescripciones mensuales de calcio en ese país.

La nueva revisión de Lewis y col. representa el máximo nivel de evidencia disponible a la fecha y deja al descubierto la fragilidad de las recomendaciones basadas en baja calidad de evidencia.

Agustín Ciapponi [ Hospital Italiano de Buenos Aires e Instituto de Efectividad Clínica y Sanitaria agustin.ciapponi@ hospitalitaliano.org.ar ] Gabriela Cormick y Jose Belizán [ Instituto de Efectividad Clínica y Sanitaria. ]

Ciapponi A, Cormick G y Belizán J.Los suplementos de calcio ¿aumentan el riesgo de eventos cardiovasculares? Evid Act Pract Ambul. 2015;18(3):8788. Jul-Sep. Comentado de: Bolland MJ, Avenell A, Baron JA y col. Effect of calcium supplements on risk of myocardial infarction and cardiovascular events: meta-analysis. BMJ. 2010 Jul 29;341:c3691. PMID: 20671013. y Lewis JR, Radavelli-Bagatini S, Rejnmark L y col. The effects of calcium supplementation on verified coronary heart disease hospitalization and death in postmenopausal women: a collaborative meta-analysis of randomized controlled trials. J Bone Miner Res. 2015 Jan;30(1):165-75. PMID: 25042841.

\section{Referencias}

1. Bolland MJ, y col. Vascular events in healthy older women receiving calcium supplementation: randomised controlled trial. Bmj. 2008;336(7638):262-266.

2. Reid IR, y col. Randomized controlled trial of calcium in healthy older women. The American joumal of medicine. 2006;119(9):777-785.

3. Hsia J, y col. Calcium/vitamin D supplementation and cardiovascular events. Circulation. 2007;115(7):846-854.

4. Guyatt G, y col. GRADE guidelines: 1. Introduction-GRADE evidence profiles and summary of findings tables. J Clin Epidemiol. 2011:64(4):383-394.

5. Cormick $\mathrm{G}$, y col. Calcium supplementation for prevention of primary hypertension. The Cochrane database of systematic reviews. 2015;6:CD010037. 